\title{
Psychological and occupational impact on healthcare workers and its associated factors during the COVID-19 outbreak in China
}

\author{
Xiaodan Zhang ${ }^{1,2} \cdot$ Ye Jiang $^{1,3} \cdot \mathrm{Hu} \mathrm{Yu}^{1,2} \cdot$ Yafen Jiang $^{1,2} \cdot$ Qiongfeng Guan $^{1,2} \cdot$ Weihe Zhao $^{1,3} \cdot$ Yingying Mao $^{1,2} \cdot$ \\ Danfeng Huang ${ }^{1,2} \cdot$ Wenke Hong ${ }^{1,2} \cdot \mathrm{Da} \mathrm{Li}^{1,2}$
}

Received: 22 June 2020 / Accepted: 30 January 2021 / Published online: 3 March 2021

(c) The Author(s), under exclusive licence to Springer-Verlag GmbH, DE part of Springer Nature 2021

\begin{abstract}
Purpose China was affected by an outbreak of coronavirus disease 2019 (COVID-19) in 2019-2020. Research data are needed to develop evidence-driven strategies to reduce the adverse psychological and occupational impacts on healthcare workers (HCWs).

Methods From March 1, 2020, to March 8, 2020, 946 HCWs in China completed a survey consist of sociodemographic data, precautionary measures against COVID-19, and concerns about COVID-19. Self-administered questionnaire were collected to assess psychological and occupational adverse outcomes of HCWs. Multivariable logistic regression analysis was performed to identify factors associated with the outcomes.

Results A total of $55.0 \%, 56.0 \%$ and $48.3 \%$ of the HCWs experienced burnout, psychological distress and posttraumatic stress, respectively. A total of seven factors were independently associated with burnout: good health status (OR $0.51,95 \%$ CI 0.36-0.71), fear of contagion (OR 1.31, 95\% CI 1.003-1.79), avoiding contact with children (OR 1.40, 95\% CI 1.03-1.91), enough staff support at the workplace (OR 0.59, 95\% CI 0.38-0.92), having to work overtime (OR 1.37, 95\% CI 1.03-1.83), maladaptive coping (OR 3.28, 95\% CI 2.42-4.45) and adaptive coping (OR 0.47, 95\% CI 0.35-0.62). A total of 11 factors were independently associated with high psychological distress: having one child (OR 0.54, 95\% CI 0.38-0.77), good health status (OR 0.57, 95\% CI 0.39-0.83), alcohol abuse (OR 1.51, 95\% CI 1.02-2.25), thinking the epidemic would continue for quite a long time (OR 1.59, 95\% CI 1.08-2.34), wearing extra-work clothes (OR 1.51, 95\% CI 1.06-2.15), effective protective equipment (OR 0.45, 95\% CI 0.22-0.90), enough staff support at the workplace (OR 0.55, 95\% CI 0.34-0.89), unable to take care of families (OR 1.99, 95\% CI 1.42-2.78), economic losses (OR 1.62, 95\% CI 1.14-2.31), maladaptive coping (OR $6.88,95 \%$ CI $4.75-9.97$ ), and adaptive coping (OR 0.29, 95\% CI 0.21-0.41). These factors were independently associated with posttraumatic stress: living with the elderly (OR 1.46, 95\% CI 1.04-2.05), alcohol abuse (OR 1.41, 95\% CI 1.002-1.98), working at a 3A hospital(OR 0.66, 95\% CI 0.49-0.88), acquaintances confirmed COVID-19 (OR 2.14, 95\% CI 1.20-3.84), fear of contagion (OR 1.87, 95\% CI 1.40-2.50), believing they would survive if infected (OR 0.63, 95\% CI 0.46-0.86), selfdisinfected after arriving home (OR 1.43, 95\% CI 1.01-2.02), interpersonal isolation (OR 1.65, 95\% CI 1.21-2.26), unable to take care of families (OR 1.41, 95\% CI 1.05-1.88) and maladaptive coping (OR 3.09, 95\% CI 2.32-4.11).

Conclusion The variance in adverse outcomes was explained by the effect of various factors, which will help policymakers better prepare for subsequent potential outbreaks of COVID-19.
\end{abstract}

Keywords Epidemic outbreaks · Questionnaire study · Health care staff · Psychologic distress $\cdot$ Burnout · Regression analysis

\section{Introduction}

Coronavirus disease 2019 (COVID-19) was first detected in Hubei province in December 2019 and spread with alarm-

$\mathrm{DaLi}$

huiyue999@gmail.com

Extended author information available on the last page of the article ing ease across the globe (Wang et al. 2020). Until June 21, 2020 , more than $66,729,375$ persons in 211 countries and areas had been affected, killing 1,535,982 of them [WHO 
Coronavirus Disease (COVID-19) Dashboard, December 08, 2020]. The COVID-19 crisis was unprecedented in terms of its infectiousness, as it quickly spread to different countries (Zhao et al. 2020). Based on current evidence, the actual route of transmission is still debatable (Huang et al. 2020). The average incubation period is estimated to be 5.2 days, with significant variation among patients, and the longest incubation period was as long as 24 days ( $\mathrm{Li}$ et al. 2020). Older men with medical comorbidities are more likely to become infected, with worse outcomes (Chen et al. 2020). Asymptomatic spread has also been reported (Chen et al. 2020; Ryu and Chun 2020). The provisional fatality rate by the WHO is approximately $2 \%$, and some researchers estimate the rate to range from 0.3 to $0.6 \%$; however, with the severe overload of medical resources, the fatality rate could skyrocket to over $10 \%$ (Nishiura et al. 2020). These characteristics may generally increase perceived risk.

In the affected countries, thousands of HCWs fighting on the frontline have attempted to quell the outbreak, but they are at great risk of contracting COVID-19 from patients. It was reported that 3019 HCWs from 422 medical institutions were infected with COVID-19, and 5 of them died in China as of February 11, 2020 (Epidemiology unit of COVID-19 emergency response mechanism 2020). In some cases, transmission to HCWs occurred even when they were wearing masks, eye protection, gowns and gloves. The everincreasing number of confirmed and suspected cases, the overwhelming workload, the depletion of the supply of personal protection equipment, the lack of specific drugs, and feelings of being inadequately supported may all contribute to the mental burden of these HCWs. It is likely that HCWs suffer from high levels of associated psychological stress and trauma. Sadly, in countries with severe outbreaks, suicide has been reported among HCWs (Nordt. 2020). Thus, a timely understanding of the mental health status of HCWs is urgently needed (Xiang et al. 2020).

Previous research has revealed a profound and wide range of psychosocial impacts on $19.3-58.6 \%$ of HCWs exposed to COVID-19 outbreaks, including high levels of burnout, stress, anxiety, and depression symptoms, which could have long-term implications (Restauri and Sheridan 2020; Salazar de Pablo et al. 2020). Several factors were identified that may have contributed to the distress experienced by HCWs during the epidemic: quarantine, fear of contagion, concern for family, extraordinary infection control precautions, job stress, interpersonal isolation, perceived stigma, and the conscription of non-specialists into infectious workplaces (Maunder et al. 2004; Restauri and Sheridan 2020; Salazar de Pablo et al. 2020). Concerns about the psychological and occupational effects of HCWs working during the COVID-19 outbreak are important, because this work involves the well-being of large numbers of HCWs. Additionally, this information has wider relevance to health systems in planning for emerging infections. To date, some studies have focused on the effect of COVID-19 on the mental health of healthcare workers. However, relatively few studies discussed the public health utility and the transferability of the findings. Our study aimed on providing an evidence-based review and recommendations for systemsbased interventions that may reduce physicians' psychological and occupational adverse outcomes.

The study was conducted in early March, towards the end of the epidemic in China, when all healthcare institutions were still on heightened alert. It appears that a clear understanding of the factors that mediate stress in HCWs facing COVID-19 is required to prepare effectively for the ongoing outbreaks of COVID-19 worldwide. This study aimed to assess the psychological and occupational impact of the COVID-19 outbreak on HCWs and to identify the risk and protective factors contributing to adverse outcomes.

\section{Methods}

\section{Ethical approval}

This study was conducted in accordance with the declaration of Helsinki. Participation was voluntary, and anonymity was assured. Participants were allowed to terminate the survey at any time they desired. The study was ethically approved by HwaMei Hospital, University of Chinese Academy of Sciences (approval number: PJ-NBEY-KY-2020-024-01).

\section{Study participants}

We adopted an observational and cross-sectional survey design to assess the HCWs' psychological and occupational response during the epidemic of COVID-19 using an anonymous online questionnaire administered from March 1, 2020, to March 8, 2020. Information about this study was posted on the work bulletin board of HwaMei Hospital, University of Chinese Academy of Sciences, and questionnaires were distributed to all willing HCWs. Participants were also encouraged to pass the questionnaires on to HCWs from other hospitals. HCWs completed the questionnaires through an online survey platform ('SurveyStar', Changsha Ranxing Science and Technology, Shanghai, China). The prevalence of burnout, psychological distress and posttraumatic stress among HCWs were about 55\%, $56 \%$ and $35 \%$, respectively, according to previous research and our pretest study (Maunder et al. 2006). $N=\frac{Z_{\alpha}^{2}}{d^{2}} p q$ was used to calculate sample size $(d=0.1 p, \alpha=0.05)$, which showed the minimum sample for burnout, psychological distress and posttraumatic stress study should be 314, 301 and 715 , respectively. We increased $20 \%$ considering the influence of confounding factors, leading to the minimum 
sample size of 855 . A total of 1099 HCWs completed the self-report questionnaire and 169 invalid questionnaire (5\%) were excluded. Ultimately, this study included $946 \mathrm{HCWs}$ from several provinces.

\section{The questionnaire data}

This survey measured sociodemographic data, occupational status, work exposure and quarantine of HCWs. Those worked in COVID-19 wards or fever clinics were defined as frontline workers. We also conducted questions about COVID-19 precautionary measures and concerns about COVID-19: fear of contagion, attitudes and practices with respect to COVID-19, interpersonal isolation, training and support from hospital, workload and impact on personal life.

\section{Simplified Coping Style Questionnaire (SCSQ)}

Adaptive coping and maladaptive coping regarding COVID19 were measured with the relevant subscales of the SCSQ (Liu and Meng 2011). The Cronbach's alpha values for the internal consistency of the use of the SCSQ, the adaptive coping subscale and the maladaptive coping subscale in this study was $0.86,0.90$ and 0.78 , respectively.

\section{Impact of Events scale-R (IES-R)}

The IES-R (Motlagh 2010) was adapted for use in this study to assess posttraumatic stress disorder symptoms (PTSS) experienced by subjects owing to the COVID-19 outbreak. A score of 20 or more was interpreted to indicate the best diagnostic accuracy of PTSS (Creamer et al. 2003). In this study, Cronbach's alpha was 0.93 for the IES-R.

\section{The exhaustion subscale of the Maslach Burnout Inventory-General Survey (MBI-GS)}

The exhaustion (EX) subscale of the MBI-GS was used to measure self-reported burnout by the HCWs. (Schaufeli 2002). In this study, a score of 2 or more was interpreted in this study to indicate burnout (Tomas et al. 2016; Zhu et al. 2016). The Cronbach's alpha for the internal consistency of the use of the EX subscale in this study was 0.947 .

\section{Kessler Psychological Distress Scale (K10)}

K10 was used to measure the levels of nonspecific psychological distress of the medical staff (Kessler et al. 2002). We used a threshold score of greater than 16 to identify the presence of psychological distress (Maunder et al. 2006; Paice et al. 2002). The Cronbach's alpha of K10 was 0.955 .

\section{Statistical analysis}

The analysis of the data was conducted in the "table one" package (Version 0.11.1) and "epiDisplay" package (Version 3.5.0.1) of $\mathrm{R}$ language version 3.6.1. Specifically, the differences in variables between groups were evaluated using the Pearson Chi-squared test for categorical variables expressed as frequencies (percentages, \%) and the Mann-Whitney $U$ test for non-normally distributed continuous variables expressed as medians (interquartile ranges, IQRs). Multivariate models were built by incorporating significant variables from Pearson Chi-squared test and Mann-Whitney $U$ test in a backward stepwise manner based on the Akaike information criterion (AIC). The effect size is expressed by the odds ratio (OR) value and the $95 \%$ confidence interval (CI).

\section{Results}

\section{Demographic characteristics}

A total of 946 valid questionnaires were analyzed from a total of 1099 eligible participants, giving an effective questionnaire rate of $86 \%$. The majority of respondents were well educated $(91.1 \% \geq$ bachelor's degree), were physicians $(56.0 \%)$, had primary professional title $(42.4 \%)$. A total of $27.1 \%$ of participants worked on the frontline, yet a relatively small portion of the HCWs reported unprotected exposure (2\%). Most HCWs (62.6\%) were from 3A hospital and $23.7 \%$ of them reported alcohol abuse. Other characteristics of study participants are shown in Tables 1 and 2 .

\section{Concerns about COVID-19}

A total of $43.4 \%$ of the HCWs feared being infected by COVID-19, but most of them (71.5\%) believed that they would survive if infected. The majority of respondents (77.3\%) predicted that the epidemic would continue for quite a long time. HCWs also took practices with respect to the COVID-19 infection such as: wearing an extra-work coat (31.9\%), self-disinfect after arriving home (78.1\%), avoiding contact with children (32\%) and patient $(68.5 \%)$, some of them even cut work hours (27.8\%). The majority of HCWs (ranging from 82.7 to $91.8 \%$ ) reported adequate training, protection, validated work arrangements and health support by the hospital and society. The work stress of the HCWs during the outbreak of COVID-19 has increased, $57.5 \%$ of the HCWs complained that they were unable to take care of their families, and $68.6 \%$ suffered economic losses (Table 3).

During the COVID-19 outbreak, HCWs chose several methods to protect themselves and relatives, including but not limited to purchasing one's own personal 


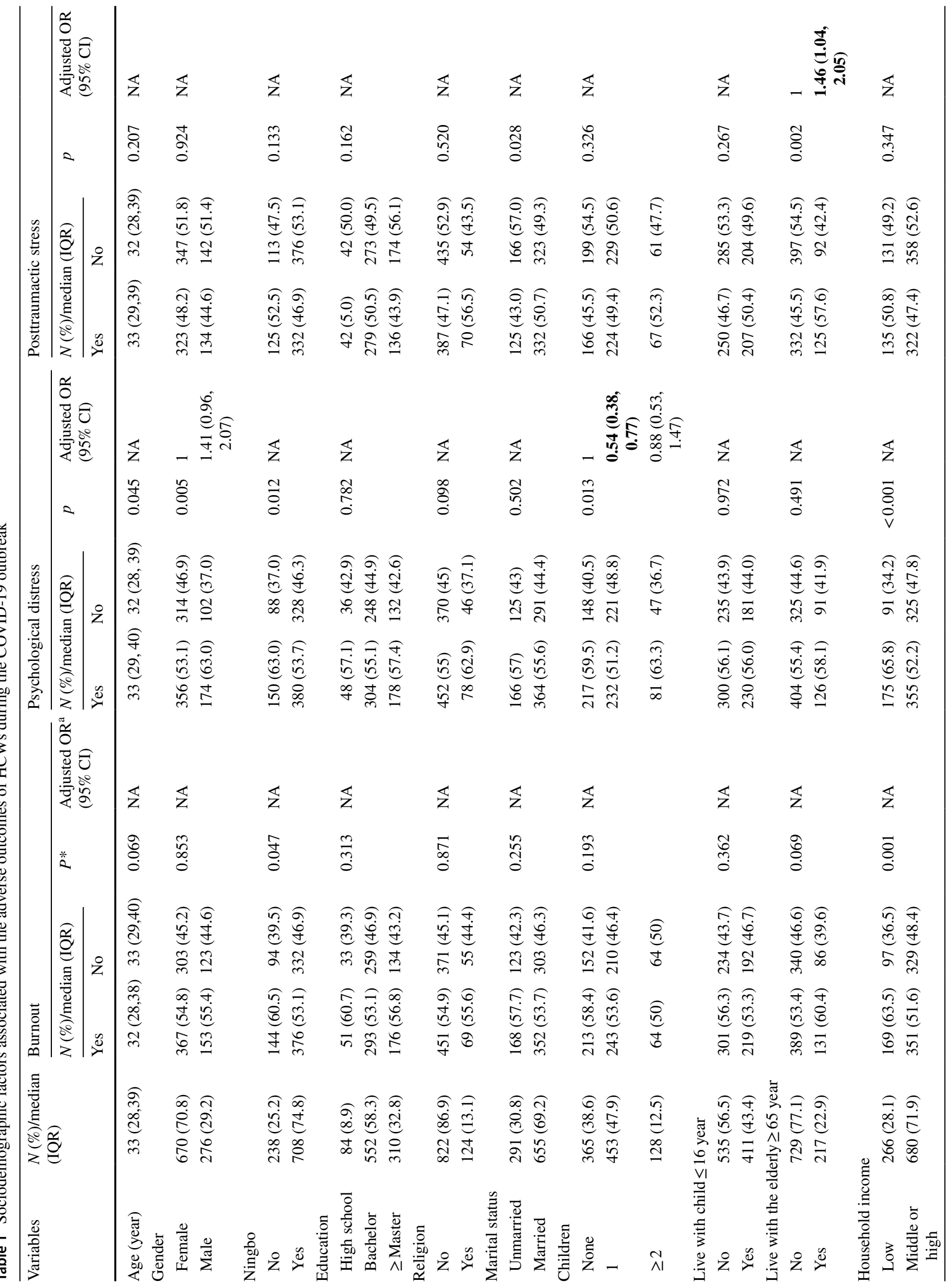




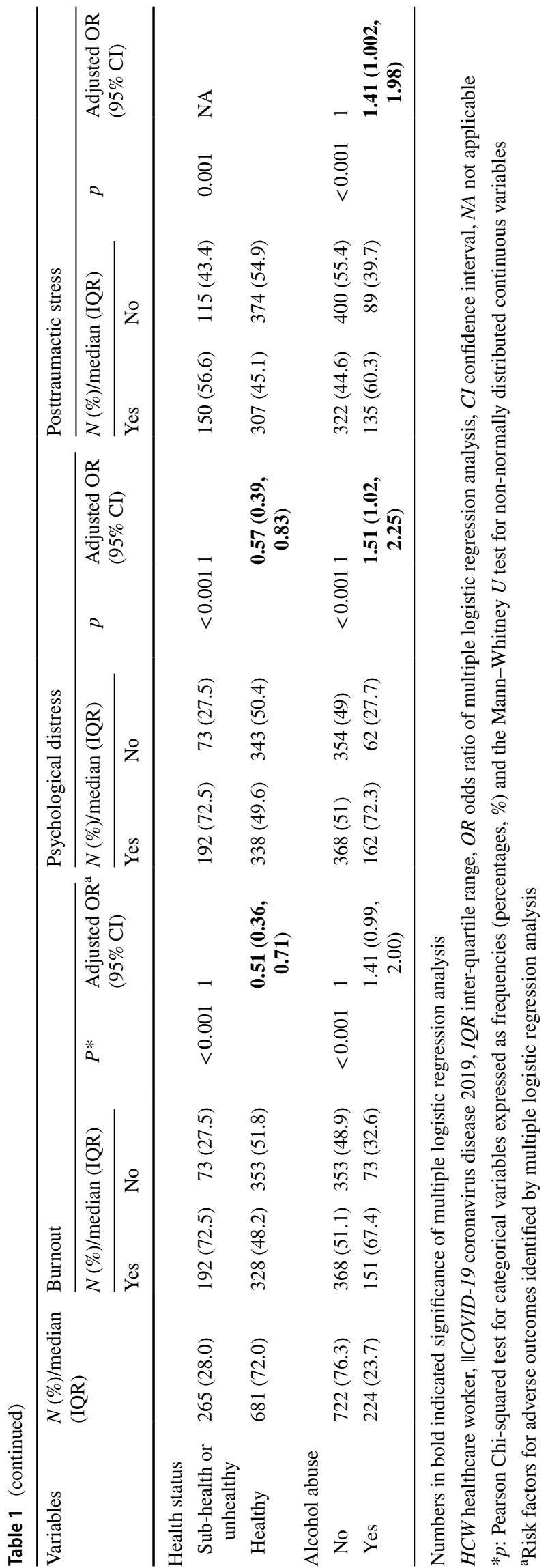

protective equipment (PPE) $(64.8 \%)$. The most common PPE were surgical masks (82.6\%) and gloves (64.1\%). Advanced PPEs were not reachable for every frontline workers; $62.5 \%$ of them were equipped with $\mathrm{N} 95$ masks, 49.2\% with goggles, $57.4 \%$ with face shields, and $79.3 \%$ with isolation gowns or protective suits. A total of $60.1 \%$ of mask users reported physical discomfort; other issues were also reported, including difficulty communicating (18.0\%) or recognizing coworkers (35.3\%), a sense of isolation (18.0\%) and skin issues (22.1\%) (Supplement Table 1).

A majority of respondents considered the accurate COVID-19 information released by state media, specific COVID-19 treatment guidelines and precautionary measures, enough PPE at the workplace and support from relatives would help them better coping with the COVID-19 outbreak. In contrast, inadequate PPE, reported cases of HCW infection or deaths led to HCWs feeling insecure. HCWs also revealed positive aspects of COVID-19 outbreak: 76.4\% participants reported an increased awareness of disease control, $14.3 \%$ of them felt an increased sense of cooperation (Supplement Table 1).

\section{Severity of measurements and associated factors}

A total of $55.0 \%, 56.0 \%$ and $48.3 \%$ of the HCWs experienced burnout, psychological distress and posttraumatic stress, respectively. Univariate analysis were conducted to identify the variables significantly associated with burnout, psychological distress and posttraumatic stress separately. Significant variables from univariate analysis were retained for stepwise regression analysis to determine which of these variables accounted for significant variance in each adverse outcome. A total of seven factors were independently associated with burnout: good health status (OR $0.51,95 \%$ CI $0.36-0.71$ ), fear of contagion (OR 1.31, 95\% CI 1.003-1.79), avoiding contact with children (OR 1.40, 95\% CI 1.03-1.91), enough staff support at the workplace (OR 0.59, 95\% CI 0.38-0.92), having to work overtime (OR 1.37, 95\% CI 1.03-1.83), maladaptive coping (OR 3.28, 95\% CI 2.42-4.45) and adaptive coping (OR 0.47, 95\% CI $0.35-0.62$ ). A total of 11 factors were independently associated with high psychological distress: having one child (OR $0.54,95 \%$ CI $0.38-0.77$ ), good health status (OR 0.57, 95\% CI 0.39-0.83), alcohol abuse (OR 1.51, 95\% CI 1.02-2.25), thinking the epidemic would continue for quite a long time (OR 1.59, 95\% CI 1.08-2.34), wearing extra-work clothes (OR 1.51, 95\% CI 1.06-2.15), effective protective equipment (OR 0.45, 95\% CI 0.22-0.90), enough staff support at the workplace (OR $0.55,95 \%$ CI 0.34-0.89), unable to take care of families (OR 1.99, 95\% CI 1.42-2.78), economic losses (OR 1.62, 95\% CI 1.14-2.31), maladaptive coping (OR 6.88, 95\% CI 4.75-9.97), and adaptive coping (OR $0.29,95 \%$ CI $0.21-0.41$ ). These factors were independently 


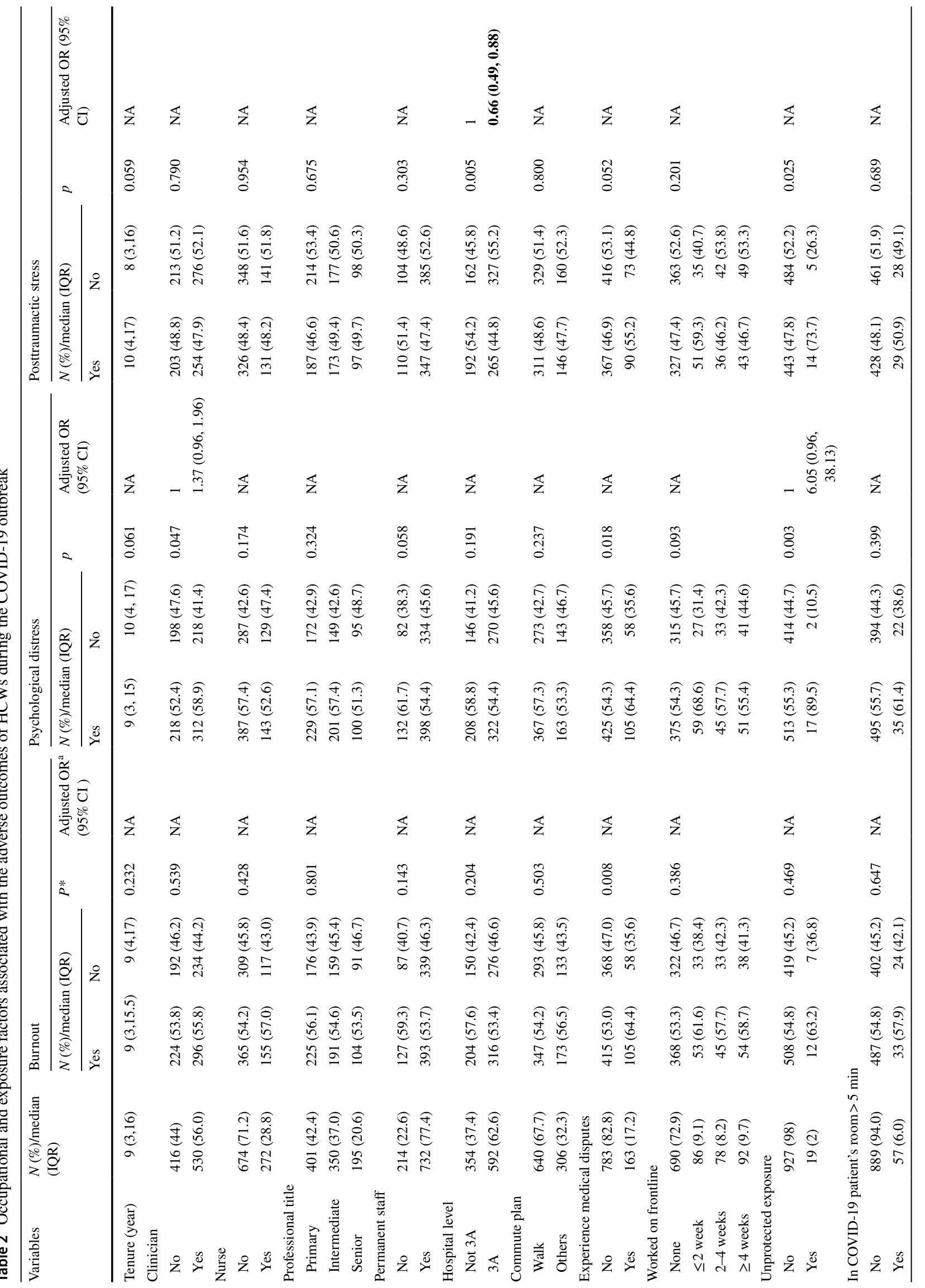




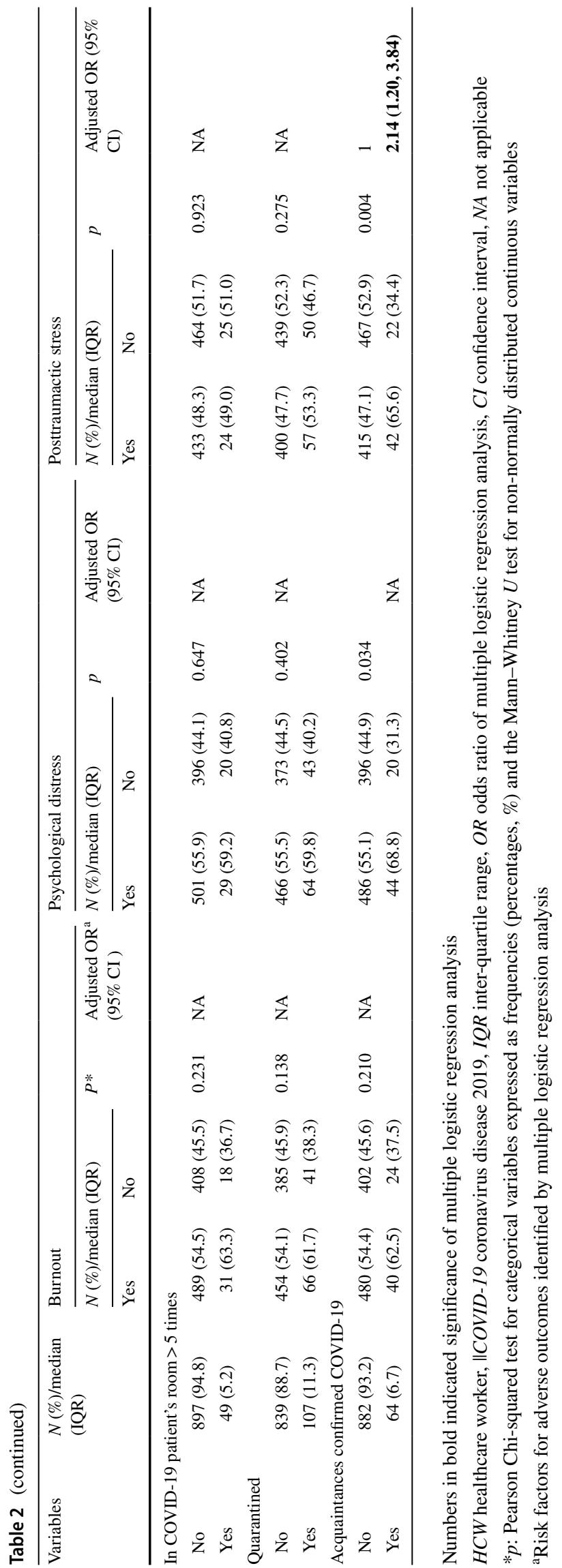

associated with posttraumatic stress: living with the elderly (OR 1.46, 95\% CI 1.04-2.05), alcohol abuse (OR 1.41, 95\% CI 1.002-1.98), working at a 3A hospital (OR 0.66, 95\% CI 0.49-0.88), acquaintances confirmed COVID-19 (OR 2.14, 95\% CI 1.20-3.84), fear of contagion (OR 1.87, 95\% CI 1.40-2.50), believing they would survive if infected (OR $0.63,95 \%$ CI 0.46-0.86), self-disinfected after arriving home (OR 1.43, 95\% CI 1.01-2.02), interpersonal isolation (OR 1.65, 95\% CI 1.21-2.26), unable to take care of families (OR 1.41, 95\% CI 1.05-1.88) and maladaptive coping (OR $3.09,95 \%$ CI 2.32-4.11). The results are shown in Tables 1 , 2 and 3.

\section{Discussion}

This cross-sectional survey enrolled 946 respondents and about half of the HCWs reported burnout, psychological distress or posttraumatic stress during the COVID-19 outbreak. Our results were similar to previously studies which revealed high rates of symptoms of burnout (19.3-53.5\%), psychological distress (28.4-56.6\%) and posttraumatic stress (13.2-58.6\%) in the in HCWs during the pandemic (Salazar de Pablo et al. 2020). The evaluation of mediating factors suggested that systemic, individual and occupational factors were associated with the adverse psychological and occupational effects of the COVID-19 outbreak. These findings could be framed in terms of their potential value for the future, and we want to learn from the COVID-19 experience in China to try to buffer this negative impact.

Health authorities need to identify high-risk groups of HCWs based on sociodemographic information for early psychological interventions. Our sociodemographic data suggested that HCWs had one child were more likely to exhibit reduced psychological distress. Multivariable logistic regression analysis showed that HCWs who lived with elderly adults during the outbreak reported greater posttraumatic stress. We attribute this to the fact that the HCWs were worried about transmitting COVID-19 to the elderly, who were more likely to become infected by COVID-19 and had worse outcomes (Chen et al. 2020). The results of our study suggested that temporary separation between HCWs and the elderly adults during the outbreak of COVID-19 (but frequent communication online) may be helpful in reducing the adverse effects. Providing effective family support for healthcare workers may help to minimize the adverse mental health issues among HCWs during epidemic. We also found that $\mathrm{HCW}$ s with a poor health status were significantly associated with psychological distress, indicating the importance of maintaining one's daily health status in the face of major infectious diseases. Hospital administrators should consider health status of HCWs when assigning work during the COVID-19 outbreak. In this study, HCWs with 


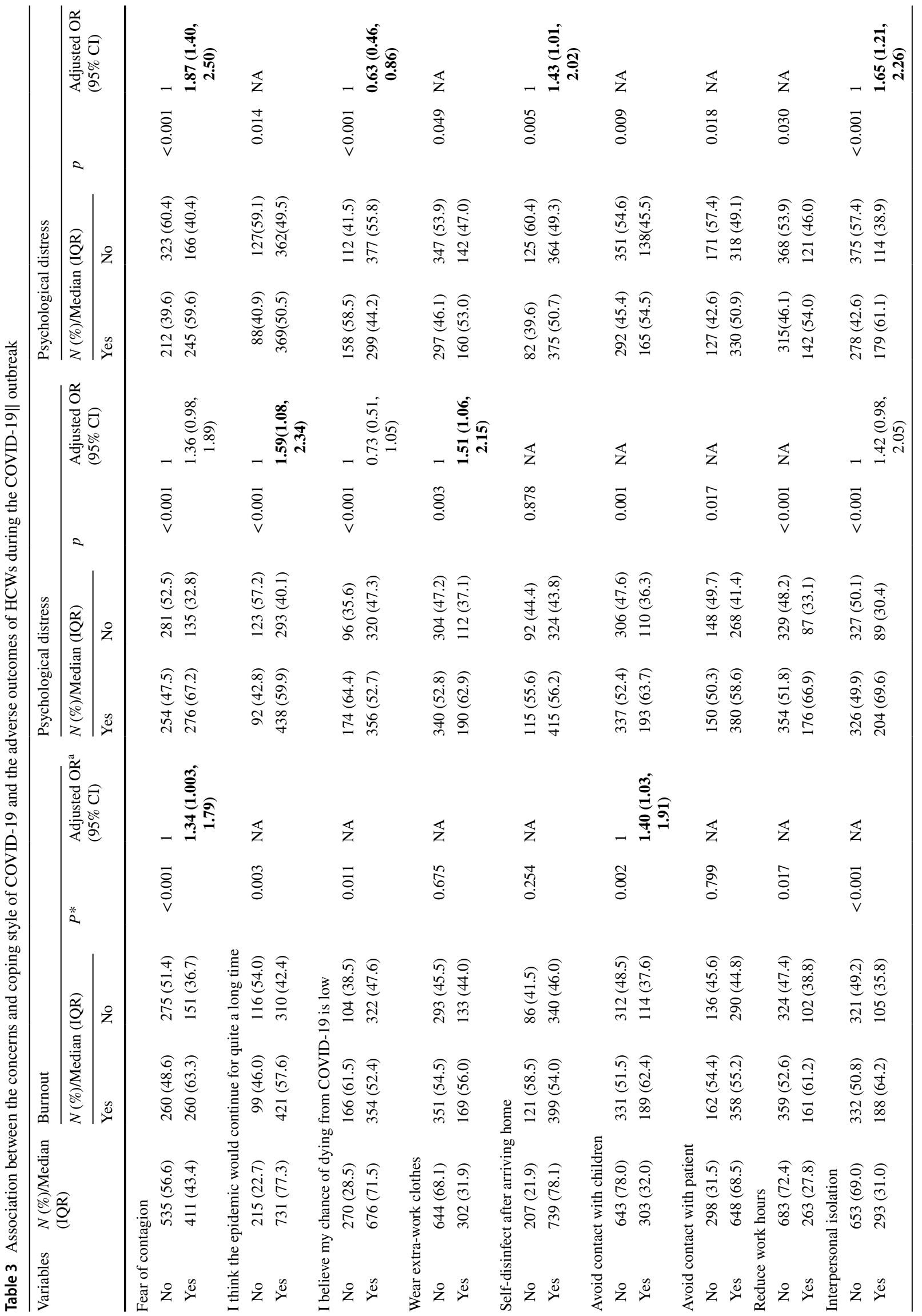




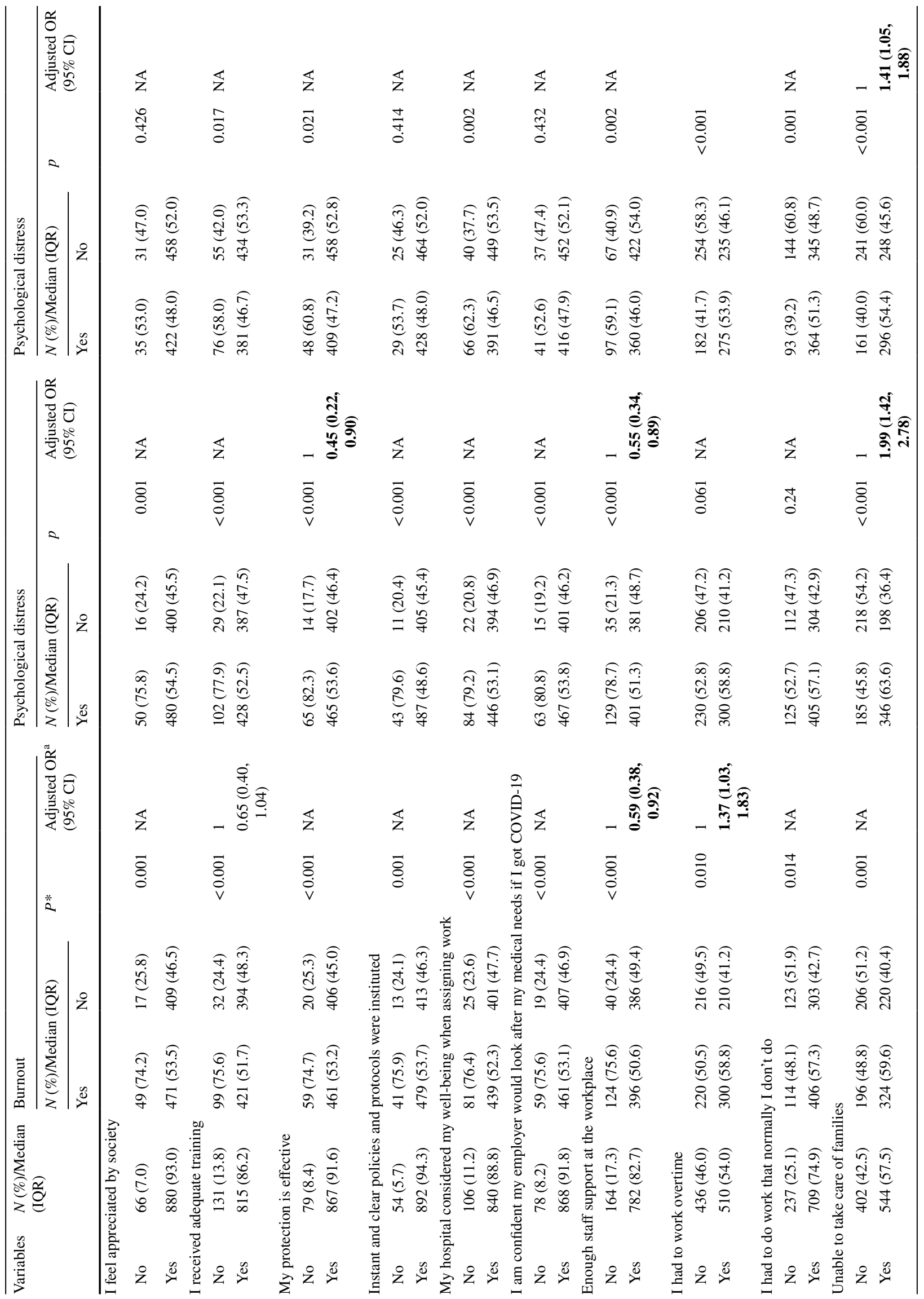




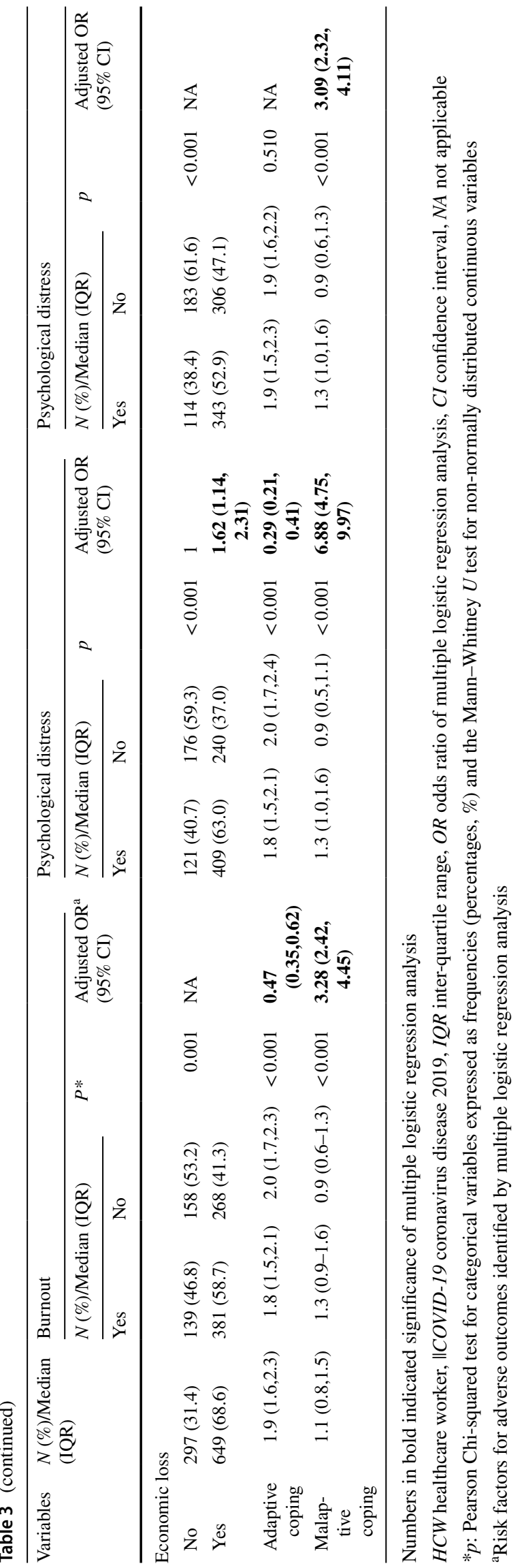

alcohol abuse were significantly associated with higher risk of psychological distress and posttraumatic stress. HCWs suffering from alcohol abuse may pose a risk for the health and safety of themselves. The occupational health specialist charged of medical surveillance of hospital workers need to recognize HCWs suffering from alcohol abuse and define effective strategies to deal with the problem such as transfer from frontline work.

)Occupational factors often have an effect on psychological outcomes. Hospital level was the factor strongly associated with posttraumatic stress. HCWs worked at $3 \mathrm{~A}$ hospital exhibited reduced posttraumatic stress. We attribute this to $\mathrm{HCWs}$ worked at $3 \mathrm{~A}$ hospital were more likely to have experience to deal with disasters and public infectious events, and most of them had lower economic stress. Currently, with the increase in the number of cases of COVID19 infection in China, frontline medical staff were required to wear protective masks and protective clothing. Many participants expressed discomfort in using PPE, especially wearing masks and isolation suits, as a highly intense shift in PPE may cause feelings of suffocation and dehydration, not to mention inconveniences with respect to urination and defecation, all of which may cause added stress. However, discomfort is incomparable to safety, and a shortage of PPE for frontline workers is realistic. In addition to isolation wards and fever clinics, personnel working in other departments may also contact COVID-19 patients, but special PPEs such as N95 mask were not provided to them by medical authorities. The protective effect of the PPEs were also doubted, as a portion of the PPE provided to hospital were not for medical use, some cautious HCWs chose to wear extrawork clothes, which would aggravate psychological distress according to our result. Cases of infection and death among $\mathrm{HCW}$ s were still reported in some hospitals, which may enhance the insecurity of HCWs, while providing enough and effective PPEs was an easy way to give HCWs security (Epidemiology Working Group for Ncip Epidemic Response and Prevention 2020). Great efforts should be made to access to abundant PPEs, including receiving donation from society, government's coordination, and medical supplies manufacturing increase.

Perceptions of risk of infection are easily understood due to COVID-19's preferential transmission to hospital workers and its substantial mortality. With the increasing reported cases of HCWs and acquaintances infection or deaths, HCWs experienced fear of getting infected, and these were significantly associated with psychological distress. HCWs feared of contagion were more likely to exhibit reduced work efficiencies and were significantly associated with burnout. Some of the HCWs predicted the epidemic would continue for quite a long time, which was the predictors of high psychological distress. Most HCWs believe they would survive if infected, which was strongly associated 
with lower posttraumatic stress. Professional psychotherapy teams should be established to support the mental health of medical staff and provide individually targeted interventions, especially for HCWs who experienced acquaintances infected or died from COVID-19. A more supportive social environment and more friendly mass media would be helpful to HCWs psychological health during an infectious disease outbreak. In China, there was a timely data in open to provide people with dynamic and important information so that they could fully understand the pandemic and policies; hospitals organized periodically training system to help HCWs updating knowledge of COVID-19. These would be helpful to reduce HCWs' fear of the uncontrollable future and reduce adverse psychological and occupational effects of the COVID-19 outbreak.

Most HCWs disinfected themselves as soon as they arrived home and some of them avoided contacting with children not to passing the virus on to their family members, which were significantly associated with burnout and posttraumatic stress. During January to March, when the outbreak was hardly under control, instead of returning home, most frontline workers were accommodated in designated hotels or dormitory, which may alleviate their concern. Most of the HCWs reported that there were adequate staff at their workplace to handle the different demands, which were independently associated with decreased burnout and psychological distress. Social isolation in infectious diseases was related to the threat of infection and reduced contact and may be a stress-provoking feature (Hall et al. 2003). Our study suggested that interpersonal isolation provoked psychological distress. Efforts to reduce the negative impact of interpersonal isolation could include creative efforts to increase effective interpersonal communication, such as web-based support or discussion groups.

Job-related stressors during the COVID-19 crisis included increased workload and a lack of control over work, including involuntary conscription. The study showed that working overtime was a significant predictor of burnout. Therefore, it is important to attend to organizational characteristics that are known to buffer burnout during the prepandemic period, which include reducing patient-to-HCW ratios, arranging HCWs' work and rest schedules reasonably and increasing perceived empowerment (Laschinger et al. 2001). An inability to take care of their family was the independent predictive factor of posttraumatic stress and psychological distress. Personal economic losses were significantly associated with psychological distress. In China, most frontline workers were transferred from other department after detailed pre-training, whose work time was no more than a month. Substantial subsidy was leaned to HCWs especially the frontline workers in China during the pandemic. Preference to the frontline workers was rewarded in professional title promotion. These supportive interventions such as increased incomes and reasonable work and rest schedules for two-worker households may be useful for HCWs.

Maladaptive coping was a strong independent risk factor for worse mental health and occupational outcomes in all dimensions of interest. Adaptive coping could significantly reduce psychological distress and burnout. According to previous studies, social support contributes to improving self-efficacy, leading to a sense of professional achievement, which improves coping mechanisms under stress (Glozah 2015; Meixia et al. 2016). For example, hostile confrontation and self-blame may be reduced in a work environment that fosters positive working relationships through effective leadership (Yank et al. 1992). It is important to encourage staff to plan for future outbreaks, reducing the tendency to cope by means of avoidant strategies and enhancing coping through problem solving and peer support. Programs directed towards healthy lifestyles, diet, exercise, and smoking cessation may also be important in providing support to staff.

After the outbreak, China tried to adopt a thorough prevention measures in an attempt to bring the virus under control. Individuals could apply or download a health QR codes in three colors-green, yellow and red-indicating different risks to serve as permits for hospital safety. Designated hospitals were organized for treatment of confirmed patients and HCWs were recruited from different departments, which would be helpful to prevent collapse of the health systems. When the lessons of COVID-19 in China applied to other affected countries, effective protection, training and support may be a primary target to bolster the resilience of HCWs who will face future outbreaks. The State Council also issued a notice to strengthen psychological crisis interventions and psychological counseling for healthcare workers. Mental health psychological centers set up psychological consultation platforms, developed psychological intervention plans, and offered remote psychological interventions to provide psychological assistance for healthcare workers.

Positive aspects of the COVID-19 outbreak were also reported. Most of the staff noted an increased awareness and experience of infection control. The majority of HCWs reported treasuring life and work more. It may be useful for preparatory training packages and interventions to encourage healthcare workers focusing on the potential positive impact of their work or considering coping strategies that may help them to see the positive effects of working in a crisis.

This study has several limitations. The most significant methodological limitation of this study was that we use subjective self-reported questionnaires online to obtain the data, which was not based on a random selection of the sample, and the study population might not reflect the actual patterns of the general population. Nonetheless, our results must be interpreted conservatively. Among the study's limitations were its small sample size, and cohort studies with larger 
samples are needed to investigate the psychological and occupational impact of COVID-19 outbreaks on HCWs. A further limitation is that self-reports of COVID-19 experiences do not provide an objective evaluation of actual differences in the training, protection, or support that HCWs received. Regardless of the limitations, the Impact of the COVID-19 Study provides a window to the long-term effects of working during times of extraordinary infectious risk.

Our findings demonstrate a significant adverse psychosocial and occupational impact of the COVID-19 outbreak in China on HCWs. The findings from this study may provide support for the implementation of measures to improve the social support of medical staff during increased demands associated with COVID-19 outbreak at this time. Future follow-up investigations using both qualitative and quantitative approaches will be necessary to understand the psychosocial effects of COVID-19 on HCWs over time.

Supplementary Information The online version contains supplementary material available at https://doi.org/10.1007/s00420-021-01657-3.

Acknowledgements The authors would like to acknowledge the volunteers who participated in the study.

Funding This study was supported by Ningbo Health Branding Subject Fund (PPXK 2018-05) and Zhejiang Provincial Natural Science Foundation of China (LGF20H090007).

\section{Compliance with ethical standards}

Conflict of interest The authors declare that they have no conflict of interest.

\section{References}

Chen N et al (2020) Epidemiological and clinical characteristics of 99 cases of 2019 novel Coronavirus pneumonia in Wuhan, China: a descriptive study. Lancet 395:507-513. https://doi.org/10.1016/ S0140-6736(20)30211-7

Creamer M, Bell R, Failla S (2003) Psychometric properties of the impact of event scale-revised. Behav Res Ther 41:1489-1496. https://doi.org/10.1016/j.brat.2003.07.010

Epidemiology Working Group for Ncip Epidemic Response and Prevention (2020) The epidemiological characteristics of an outbreak of 2019 novel Coronavirus Diseases (COVID-19) in China. Zhonghua Liu Xing Bing Xue Za Zhi 41:145-151. https://doi. org/10.3760/cma.j.issn.0254-6450.2020.02.003

Glozah FN (2015) Exploring Ghanaian adolescents' meaning of health and wellbeing: a psychosocial perspective. Int J Qual Stud Health Well-being 10:26370. https://doi.org/10.3402/qhw.v10.26370

Hall LM, Angus J, Peter E, O’Brien-Pallas L, Wynn F, Donner G (2003) Media portrayal of nurses' perspectives and concerns in the SARS crisis in Toronto. J Nurs Scholarsh 35:211-216. https ://doi.org/10.1111/j.1547-5069.2003.00211.x

Huang C et al (2020) Clinical features of patients infected with 2019 novel Coronavirus in Wuhan. China Lancet 395:497-506. https ://doi.org/10.1016/S0140-6736(20)30183-5
Kessler R, Barker P, Colpe L, Epstein J, Zaslavsky AJPM (2002) Short screening scales to monitor population prevalences and trends in non-specific psychological distress. Psychol Med 32(6):959-976

Laschinger HK, Finegan J, Shamian J, Wilk P (2001) Impact of structural and psychological empowerment on job strain in nursing work settings: expanding Kanter's model. J Nurs Adm 31:260 272. https://doi.org/10.1097/00005110-200105000-00006

Li Q et al (2020) Early transmission dynamics in Wuhan, China, of novel Coronavirus-infected pneumonia. N Engl J Med 382:1199_ 1207. https://doi.org/10.1056/NEJMoa2001316

Liu X, Meng C (2011) Preliminary Study on the Relationship of University Students' Mindfulness with Self-concept and Coping Style. China J Health Psychol 01:92-101. https://doi.org/10.13342 /j.cnki.cjhp.2011.01.037

Maunder RG et al (2004) Factors associated with the psychological impact of severe acute respiratory syndrome on nurses and other hospital workers in Toronto. Psychosom Med 66:938-942. https ://doi.org/10.1097/01.psy.0000145673.84698.18

Maunder RG et al (2006) Long-term psychological and occupational effects of providing hospital healthcare during SARS outbreak. Emerg Infect Dis 12:1924-1932. https://doi.org/10.3201/eid12 12.060584

Meixia Z et al (2016) The impact of social support on optimistic tendency of college students: the mediating efects of sense of coherence and resilience. J Psychol Sci 2:371-376. https://doi. org/10.16719/j.cnki.1671-6981.20160218

Motlagh H (2010) Impact of event scale-revised. J Physiother 56:203. https://doi.org/10.1016/s1836-9553(10)70029-1

Nishiura $\mathrm{H}$ et al (2020) The rate of underascertainment of novel Coronavirus (2019-nCoV) infection: estimation using Japanese passengers data on evacuation flights. J Clin Med 9:419. https://doi. org/10.3390/jcm9020419

Nordt WKC (2020) COVID-19, unemployment, and suicide. Lancet Psychiatry 7:389-390. https://doi.org/10.1016/S2215 $-0366(20) 30141-3$

Paice E, Rutter H, Wetherell M, Winder B, Mcmanus ICJME (2002) Stressful incidents, stress and coping strategies in the pre-registration house officer year. Med Educ 36:56-65

Restauri N, Sheridan AD (2020) Burnout and posttraumatic stress disorder in the Coronavirus Disease 2019 (COVID-19) pandemic: intersection, impact and interventions. J Am Coll Radiol 17:921926. https://doi.org/10.1016/j.jacr.2020.05.021

Ryu S, Chun BC, Korean Society of Epidemiology -nCo VTFT (2020) An interim review of the epidemiological characteristics of 2019 novel coronavirus. Epidemiol Health 42:e2020006. https://doi. org/10.4178/epih.e2020006

Salazar de Pablo G et al (2020) Impact of coronavirus syndromes on physical and mental health of health care workers: systematic review and meta-analysis. J Affect Disord 275:48-57. https://doi. org/10.1016/j.jad.2020.06.022

Schaufeli WB, Salanova M, González-romá V, Bakker AB (2002) The measurement of engagement and burnout: a two sample confirmatory factor analytic approach. J Happiness Stud 3:71-92

Tomas JM, de Los SS, Alonso-Andres A, Fernandez I (2016) Validation of the maslach burnout inventory-general survey on a representative sample of dominican teachers: normative data. Span J Psychol 19:E83. https://doi.org/10.1017/sjp.2016.91

Wang C, Horby PW, Hayden FG, Gao GF (2020) A novel coronavirus outbreak of global health concern. Lancet 395:470-473. https:// doi.org/10.1016/S0140-6736(20)30185-9

WHO Coronavirus Disease (COVID-19) Dashboard. https://covid 19.who.int/table. Accessed 08 Dec 2020

Xiang YT, Yang Y, Li W, Zhang L, Zhang Q, Cheung T, Ng CH (2020) Timely mental health care for the 2019 novel coronavirus outbreak 
is urgently needed. Lancet Psychiatry 7:228-229. https://doi. org/10.1016/S2215-0366(20)30046-8

Yank GR, Barber JW, Hargrove DS, Whitt PD (1992) The mental health treatment team as a work group: team dynamics and the role of the leader. Psychiatry 55:250-264. https://doi.org/10.1080/00332 747.1992 .11024598

Zhao $S$ et al (2020) Estimating the unreported number of novel Coronavirus (2019-nCoV) cases in China in the first half of January 2020: a data-driven modelling analysis of the early outbreak. J Clin Med 9(2):388. https://doi.org/10.3390/jcm9020388
Zhu H-Y et al (2016) Job burnout and related inluencing factors in family physicians in minhang district of shanghai. J Environ Occup Med 08:731-735. https://doi.org/10.13213/j.cnki. jeom.2016.15527

Publisher's Note Springer Nature remains neutral with regard to jurisdictional claims in published maps and institutional affiliations.

\section{Authors and Affiliations}

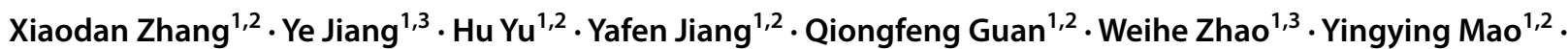 Danfeng Huang ${ }^{1,2} \cdot$ Wenke Hong ${ }^{1,2} \cdot \mathrm{Da} \mathrm{Li}^{1,2}$ (D)}

1 Department of Neurology, HwaMei Hospital, University of Chinese Academy of Sciences, Xibei Rd\#41, Ningbo 315010, Zhejiang, China

2 Ningbo Institute of Life and Health Industry, University of Chinese Academy of Sciences, Xibei Rd\#41, Ningbo 315010, Zhejiang, China
Department of Respiratory, HwaMei Hospital, University of Chinese Academy of Sciences, Xibei Rd\#41, Ningbo 315010, Zhejiang, China 